\title{
Um caso de adenite por BCG
}

Marta Sousa Tavares, * Inês Figueiredo**

\section{RESUMO}

Introdução: O Plano Nacional de Vacinação recomenda a vacina contra a tuberculose com o bacilo de Calmette e Guérin (BCG) em todos os recém-nascidos, excepto se existirem contra-indicações específicas. A sua principal complicação é a adenite (BCGite) e surge nos primeiros 6 meses após a vacinação.

Descrição do Caso: Lactente de termo que, na consulta de vigilância dos 2 meses de idade, ao exame objectivo apresentava adenite axilar esquerda, sendo o restante exame objectivo normal. Feito o diagnóstico de BCGite, referenciou-se ao Serviço de Urgência para observação e orientação, onde foi recomendado tratamento conservador. Dadas as dimensões exuberantes da adenopatia, a presença de sinais inflamatórios e as queixas do lactente, a mãe praticamente todos os dias recorreu ao centro de saúde para nos mostrar a evolução da adenite e questionar se nada mais havia a fazer para além do analgésico. A adenite foi evoluindo e ao final de 3 semanas apresentava flutuação, acabando por drenar espontaneamente, com remissão dos sintomas. Actualmente, a criança com 18 meses de idade apresenta uma cicatriz na axila esquerda.

Comentário: Dada a raridade destes casos pretendemos alertar os médicos de família para o carácter benigno da BCGite. A sua evolução é na maioria das vezes favorável e o tratamento é conservador. A referenciação só é necessária se houver complicações da adenite para avaliação de necessidade de punção ou excisão cirúrgica. Pode ser difícil tranquilizar os pais perante uma situação clínica tão exuberante e dolorosa, não havendo nenhum tratamento imediato para resolver a situação para além da atitude expectante.

Termos MeSH: BCG Vaccine; Lymphadenitis.

\section{INTRODUÇÃO}

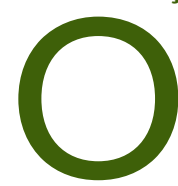

Plano Nacional de Vacinação recomenda a vacina contra a tuberculose com o bacilo de Calmette e Guérin (BCG) em todos os recém-nascidos, excepto se existirem contra-indicações específicas. ${ }^{1}$

As complicações da BCG são raras (<1:1000 vacinados). ${ }^{2}$ Uma das complicações mais comuns da BCG é a adenite (BCGite), definida como o aumento de tamanho de um gânglio linfático axilar, supraclavicular ou cervical $(\geq 2 \mathrm{~cm})$, ipsilateral ao local de inoculação da BCG, dentro dos 6 meses após a vacinação BCG. ${ }^{2-5}$

Os factores de risco para a adenite pós-vacinal dependem da idade de vacinação, da resposta imunológica, da via de administração, da técnica utilizada, da estirpe e da dose. ${ }^{1,3-4}$

Duas formas de apresentação da BCGite podem ser reconhecidas na sua evolução natural. A BCGite não-supurativa ocorre mais precocemente e normalmente resolve-se espontaneamente dentro de poucas semanas, sem quaisquer sequelas. Em alguns casos, o gânglio afectado aumenta progressivamente de tamanho e desenvolve supuração, edema e eritema da pele subjacente. ABCGite su-

* Médica Interna de Medicina Geral e Familiar, UCSP Anadia III

**Médica Assistente de Medicina Geral e Familiar, USF Alpha, Válega purativa pode desenvolver-se após 2-4 meses da vacinação da BCG e persiste por vários meses. ${ }^{1-2,5}$

O diagnóstico desta patologia é clínico. Perante uma criança previamente saudável, filha de mãe seronegativa para o HIV e com boa progressão estato-ponderal, o achado de adenite loco-regional ipsilateral ao local de inoculação da BCG, sem outra causa identificável e na ausência de febre e/ou sintomas constitucionais, é suficiente para o diagnóstico. Os exames complementares de diagnóstico não estão indicados de forma rotineira. ${ }^{2-4}$

$\mathrm{O}$ tratamento da adenite por BCG ainda se mantém controverso e não é consensual. Alguns estudos, pelo carácter benigno da evolução da BCGite não-supurativa, sugerem um tratamento conservador, enquanto na BCGite supurativa aconselham a aspiração por agulha fina de forma a encurtar o tempo de resolução e prevenir complicações como a formação de locas. A excisão cirúrgica apenas está indicada nas situações de abcessos multiloculados. $^{3-6}$ Outros autores defendem uma atitude expectante perante uma BCGite supurativa, na ausência de doença disseminada. ${ }^{2}$ A terapêutica farmacológica com anti-tuberculostáticos e/ou antibióticos não está indicada, uma vez que a medicação não altera o curso da adenite e pode levar a reacções adversas medicamentosas. ${ }^{2-6}$

Apesar de raras, as complicações da BCGite existem e 


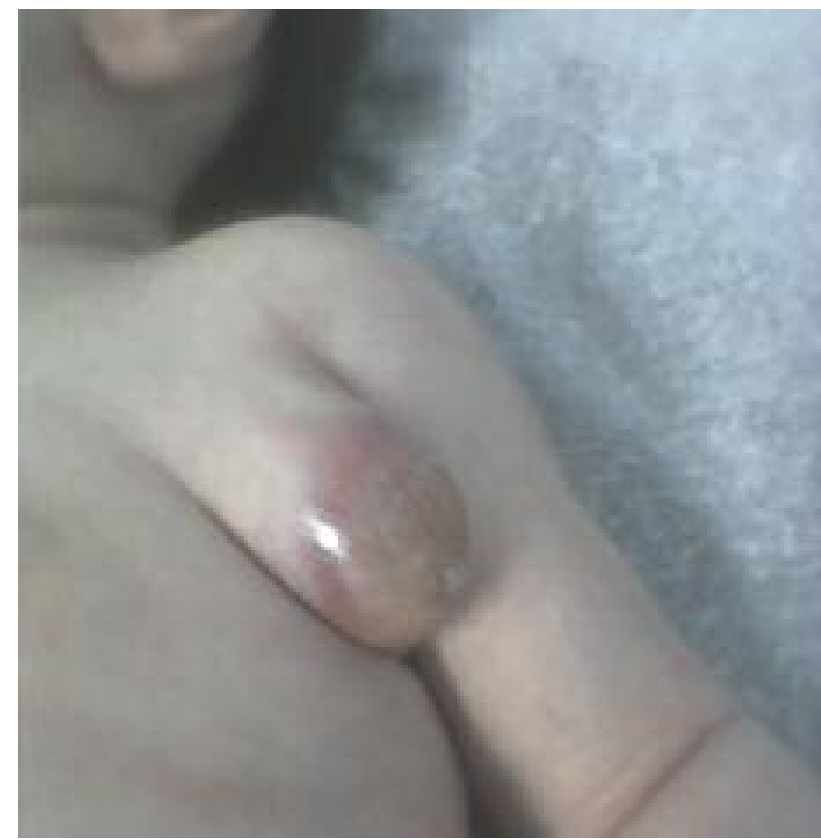

Figura 1. BCGite supurativa em lactente de 2 meses de idade.

são um desafio para o médico de família. O caso que se segue retrata um caso de BCGite no qual o desafio para o médico de família esteve na dificuldade em tranquilizar a mãe da criança acerca da benignidade da patologia.

\section{DESCRIÇÃO DO CASO}

Relatamos o caso de uma lactente de termo, caucasiana, nascida de parto eutócico, com Apgar 9/10. Apresentava um peso à nascença de 2,960 Kg e 48,5 cm de estatura. Encontra-se inserida numa família nuclear na fase II do ciclo de Duvall, da classe média alta segundo o índice de Graffar.

Na consulta de vigilância dos 2 meses, ao exame objectivo, a lactente apresentava-se um pouco irritada e queixosa. Apresentava uma adenite axilar esquerda com dimensões $4,1 \mathrm{~cm} \mathrm{x} \mathrm{2,3} \mathrm{cm} \mathrm{(figura} \mathrm{1),} \mathrm{sendo} \mathrm{o} \mathrm{restante}$ exame físico normal. Colocou-se como hipótese de diagnóstico estarmos perante uma adenite por BCG (BCGite) e referenciou-se ao Serviço de Urgência (SU) para observação e orientação. No SU confirmou-se o diagnóstico de BCGite supurativa e foi recomendado tratamento conservador. Foi medicada com paracetamol para alívio da dor, indicação de reforço da hidratação cutânea e aguardar pela drenagem espontânea.

Dado a criança se manter queixosa e irritada, a mãe, preocupada e ansiosa com a situação clínica da filha, recorreu novamente à nossa consulta e questionou se não haveria algo mais a fazer para além do paracetamol. Foi explicado à mãe que teria de aguardar pela drenagem espontânea da adenite e que, quando ocorresse, a dor iria aliviar e o quadro resolver-se-ia naturalmente. Entretanto, reforçamos a ideia de que deveria manter o paracetamol e hidratar a pele. Dado a mãe se encontrar bastante ansiosa, foi incentivada a recorrer à consulta sempre que necessário para observarmos a criança, de forma a garantir que a adenite estaria a seguir o seu curso natural de resolução ou detectar alguma complicação com necessidade de referenciação. Como tal, a mãe recorreu ao Centro de Saúde uma média de 3 vezes por semana, o que a tranquilizava.

Finalmente, após 3 semanas do início do quadro clínico, a adenite drenou espontaneamente, com alívio sintomático, ficando a mãe mais tranquila (figura 2). A drenagem da adenite durou cerca de 7 semanas.

Actualmente, a menina, com 18 meses de idade, apresenta uma cicatriz quelóide subaxilar esquerda (figura 3).

\section{COMENTÁRIO}

Dada a raridade destes casos pretendemos alertar os médicos de família para o carácter benigno da BCGite. Apesar de neste caso a criança apresentar uma adenite supurativa, para a qual alguns autores aconselham a punção aspirativa de forma a encurtar o tempo de resolução e prevenir complicações, optou-se por um tratamento conservador, como é defendido por outros autores e por aguardar pela drenagem espontânea, tendo tido um bom resultado pois, ao fim de 10 semanas do início do quadro clínico, ocorreu resolução completa sem complicações. Em alguns casos, em que é realizada a punção aspirativa para drenagem, é necessária mais do que uma aspiração, o que condiciona um prolongamento do quadro clínico e aumenta o risco de complicações. ${ }^{4}$

Dado o tratamento da BCGite ser ainda controverso, pela experiência deste caso clínico, os autores recomendam que, perante um lactente que apresente BCGite, quer seja não-supurativa como supurativa, com boa evolução, o tratamento deverá ser conservador, não havendo necessidade de referenciação. Deve ser explicado aos pais que apesar do seu aspecto exuberante, com sinais inflamatórios e dor, está indicado apenas reforço da hidratação cutânea e analgesia para alívio das dores. 


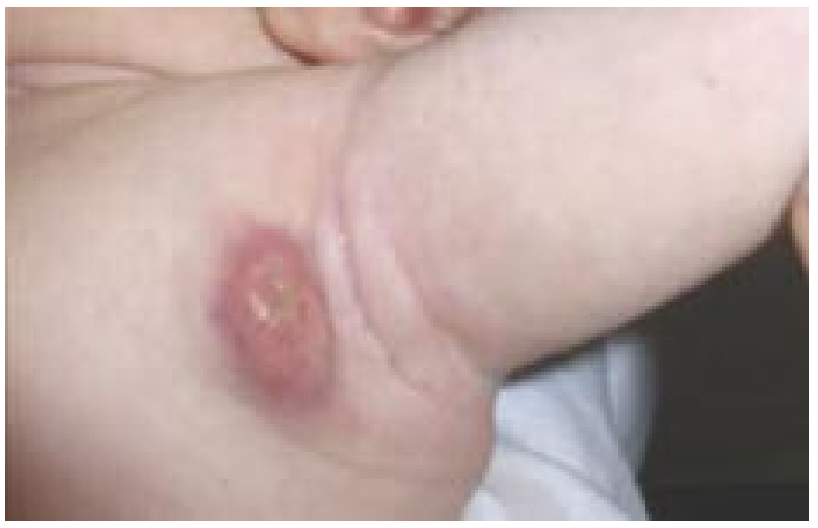

Figura 2. Aspecto da lesão de BCGite Supurativa após drenagem espontânea.

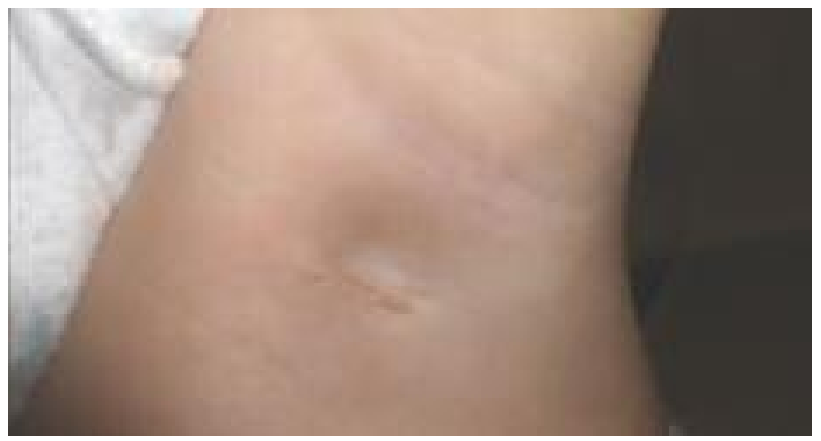

Figura 3. Cicatriz sequela de BCGite Supurativa.

\section{REFERÊNCIAS BIBLIOGRÁFICAS}

1. Direcção-Geral da Saúde. Programa nacional de vacinação: circular normativa ${ }^{\circ}$ 040/2011, de 21/12/2011. Lisboa: DGS.

2. Constatant C, Figueiredo A, Brito MJ. Adenite do bacilo CalmetteGuérin: protocolo diagnóstico e terapêutico [Bacille Calmette-Guérin adenitis: diagnostic and therapeutic guidelines]. Acta Med Port. 2011;24(S2):263-8. Portuguese

3. Govindarajan KK, Chai FY. BCG adenitis: need for increased awareness. Malays J Med Sci. 2011;18(2):66-9.

4. Goraya J,VirdiV. Bacille Calmette-Guérin lymphadenitis. Postgrad Med J. 2002;78(920):327-9.

5. Singla A, Singh S, Goraya JS, Radhika S, Sharma M. The natural course of nonsuppurative Calmette-Guérin bacillus lymphadenitis. Pediatr Infect Dis J. 2002;21(5):446-8.

6. Ali S, Almoudaris M. BCG lymphadenitis.Arch Dis Child. 2004;89(9):812.

\section{CONFLITOS DE INTERESSE}

Os autores declaram não possuir qualquer tipo de conflitos de interesse.

\author{
ENDEREÇO PARA CORRESPONDÊNCIA \\ Marta Rafaela Sousa Tavares \\ Rua das Fontainhas, $n^{\circ} 146$ \\ 3880-283 Ovar \\ Email: martarafatavares@hotmail.com
}

Recebido em 28-01-2014

Aceite para publicação em 17-09-2014

\section{ABSTRACT}

\section{ADENITIS DUE TO BCG VACCINE: A CASE REPORT}

Introduction: The Portuguese national vaccination plan recommends the Bacillus Calmette-Guérin (BCG) vaccine against tuberculosis for all newborn babies, except if there are specific contraindications. The main complication of BCG vaccine is lymphadenitis that can occur within 6 months after vaccination.

Case description: A two-month old infant born after a full-term pregnancy presented at a routine consultation with left axillary lymphadenitis. The rest of the physical examination was normal. A diagnosis of BCG lymphadenitis diagnosis was made and the patient was referred to the local emergency service for observation. Conservative treatment was recommended. Faced with large lymphadenopathy, signs of inflammation, and the baby's distress, the mother made frequent visits to the health center to show the evolution of the adenitis and to ask whether anything else could be done, aside from the administration of the analgesics. The child presented again with fluctuation and spontaneous drainage of the lymph nodes after 3 weeks. Eventually all symptoms resolved. The child was seen at 18 months old of age with a healed scar in the left axilla.

Commentary: This case demonstrates the benign nature of BCG lymphadenitis. The vast majority of cases require conservative treatment. Referral is necessary only if there are complications that might require aspiration or surgical drainage. It can be difficult to reassure parents when presented with such a striking condition and the recommended course of action is analgesia and patience, as symptoms resolve spontaneously.

MeSH terms: BCG Vaccine; Lymphadenitis. 